\title{
Mosaic crystals leading a new route to achieve ultrahigh thermoelectric performance
}

\section{Yi Xie}

Solid-state thermoelectric technology uses electrons or holes as the working fluid for heat pumping and power generation. Adopting the technology in harvesting solar heat, converting waste industrial heat into electricity, and pumping out operational heat has tremendous potential in solid-state electronics applications. A combination of highly efficient electronic transport and low heat conductivity is a prerequisite for excellent thermoelectric performance. As these two requirements are substantially orthogonal, their synchronous realization is difficult in practice, hindering the commercial use of thermoelectricity $[1,2]$.

Scientists from the Shanghai Institute of Ceramics, Chinese Academy of Sciences, in collaboration with scientists from Northwestern University and the University of Michigan, have developed a new route to realize ultrahigh thermoelectric performance by employing the concept of nanoscale mosaicity in a single-phase material. Mosaic crystals, because of certain characteristic features, is an unprecedented example for the subtle tuning of thermoelectric transports, leading to significantly improved performance (Fig. 1), as elucidated by a paper recently published in Advanced Materials [3]. The research offers a cutting-edge opportunity for studying thermoelectric transports relevant to an innovative strategy for developing high-efficiency thermoelectric materials.

Current bulk materials with the thermoelectric figure of merit $z T\left(z T=S^{2} \sigma T / \kappa\right.$, where $S$ is the Seebeck coefficient, $\sigma$ is the electrical conductivity, $T$ is the absolute temperature, and $\kappa$ is the thermal conductivity) above 2 have only been reported in single crystals [4], nanocomposites [5], and critical phase transitions [6]. The single crystal and nano-materials are two extreme crystallization regimes. Single crystals have excellent electrical and thermal conductivity because of the absence of boundary or interface scattering. In contrast, nano-materials usually have low electron and phonon conduction because the scattering on boundaries and interfaces is extremely effective.
Many groups have been developing various strategies for enhancing the performance of thermoelectric materials $[1,2]$. The most notable result reported was the large reduction in thermal conductivity without severely affecting electrical transport. However, electrical transport is usually greatly affected at the same time. In this case, any further enhancement in thermoelectric performance must originate from a balance between the electrical and thermal transports in a material.

In the paper published in Advanced Materials by Shi's and Chen's group from the Shanghai Institute of Ceramics [3], a new mosaic-crystal microstructure was proposed to balance the electrical and thermal transports. The lattice translations throughout mosaic crystals have a high degree of perfection [7]. The crystal is composed of mosaic blocks. Although each block is a perfect crystal, it is titled or rotated with respect to others by less than a minute of an arc. In contrast to ordinary polycrystals or nanocrystal materials, which have random orientations, a mosaic crystal has blocks that exhibit a nearly identical orientation. Consequently, from a macroscopic point of view, the bulk material has the appearance of a single crystal. However, it contains a number of small-angle boundaries, as shown in Fig. 1. In a mosaic crystal, excellent electronic transport, similar to that in a single crystal, occurs because of the high lattice coherence, but grain boundaries still show effective misorientation by a slight amount in scattering heat-conducting phonons, resulting in exceptional thermoelectric properties. Thus, the mosaic crystal structures can combine the characters of single crystals and nano-materials, which is very helpful for thermoelectric applications.

In mosaic crystals, the free transfer of electrons occurs along quasi-single-crystal frames, whereas the strong scattering of phonons occurs through lattice strains or mosaicnano-grain interfaces. Glass-like ultralow thermal conductivity, which is very surprising and interesting, has been reported in a conventional crystal material [3]. In addition,

Hefei National Laboratory for Physical Sciences at Microscale, Collaborative Innovation Center of Chemistry for Energy Materials, University of Science \& Technology of China, Hefei 230026, China

Email: yxie@ustc.edu.cn 

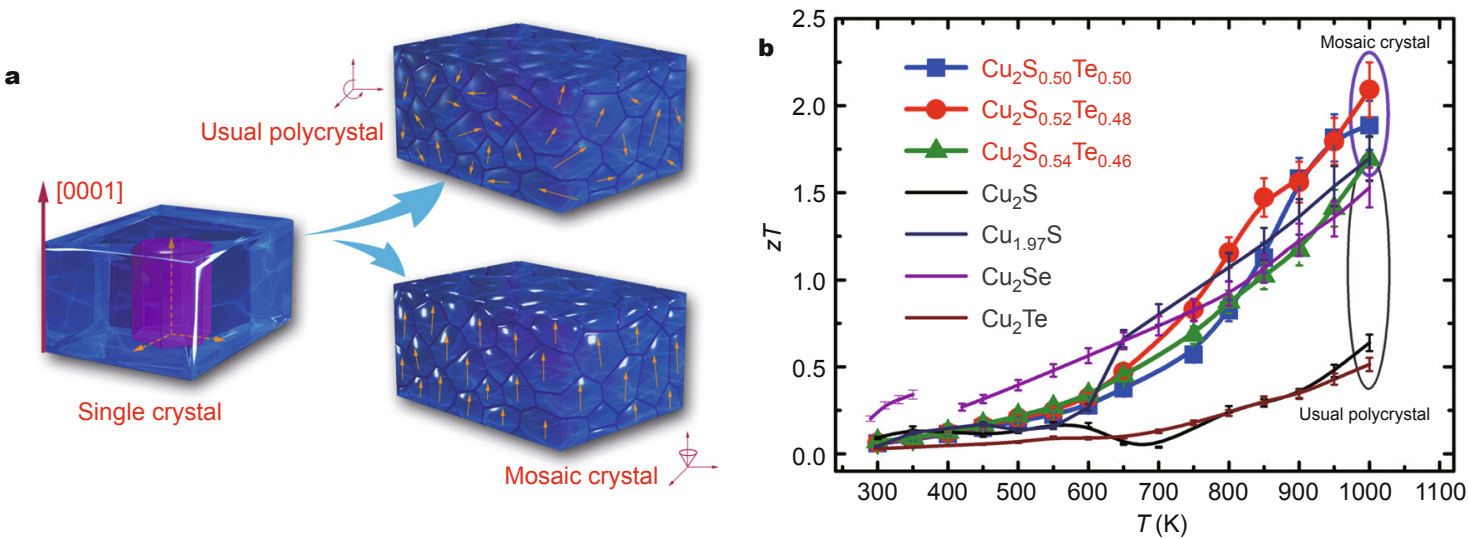

Figure 1 Enhanced $z T$ values in mosaic crystals. (a) Structural characteristics of a single crystal, a normal polycrystal, and a mosaic crystal. Arrows indicate the alignment of crystal grains. (b) Temperature dependence of $z T$ values for $\mathrm{Cu}_{2}(\mathrm{~S}, \mathrm{Te})$ mosaic crystals. Reprinted with permission from Ref. [3], Copyright 2015, Wiley.

the electron effective mass and Seebeck coefficient have also been greatly enhanced because of the additional effect of the low-angle boundaries. Thus, the simultaneous optimization of electron and phonon transports is promoted to achieve ultrahigh thermoelectric performance with the figure of merit above 2 [3].

Using nanoscale mosaicity is a step forward from the previous approaches because it enables thermoelectric properties to be tuned by multiform effects. It is expected this strategy, with the use of a flash cooling technique or a mixture of elements with vastly mismatched atomic sizes to produce mosaic nano-crystals, to be applicable to all bulk thermoelectric materials. The work probably opens a new window of realizing ultrahigh thermoelectric performance. This strategy is also expected to be easily applied in conjunction with existing successful approaches such as band-structure engineering to enhance the thermoelectric performance further.
Received 9 June 2015; accepted 10 June 2015; published online 19 June 2015

1 Bell LE. Cooling, heating, generating power, and recovering waste heat with thermoelectric systems. Science, 2008, 321: 1457-1461

2 Snyder GJ, Toberer ES. Complex thermoelectric materials. Nat Mater, 2008, 7: 105-114

3 He Y, Lu P, Shi X, et al. Ultrahigh thermoelectric performance in mosaic crystals. Adv Mater, doi: 10.1002/adma.201501030

4 Zhao LD, Lo SH, Zhang Y, et al. Ultralow thermal conductivity and high thermoelectric figure of merit in SnSe crystals. Nature, 2014, 508: 373-377

5 Biswas K, He J, Blum ID, et al. High-performance bulk thermoelectrics with all-scale hierarchical architectures. Nature, 2012, 489: 414-418

6 Liu H, Yuan X, Lu P, et al. Ultrahigh thermoelectric performance by electron and phonon critical scattering in $\mathrm{Cu}_{2} \mathrm{Se}_{1-x} \mathrm{I}_{x}$. Adv Mater, 2013, 25: 6607-6612

7 Darwin CG. The reflexion of X-rays from imperfect crystals. Philos Mag, 1922, 43: 800-829 